\title{
Clinical Examinations of Thymic Abnormalities and Significance of Thymectomy in Patients with Myasthenia Gravis and Other Autoimmune Diseases
}

\author{
Hiroshi Yoshimatsu, Yoshiya Ishikura, Masaru Murakami and \\ Hideyuki KAWAHARA \\ Department of Thoracic and Cardiovascular Surgery, School of Medicine, University of \\ Occupational and Environmental Health, Kitakyushu 807, Japan
}

\begin{abstract}
Recent advances in the study of immunity and its allied fields have disclosed that the thymus, as one of the central lymphoid tissues, plays an important role in immune regulation. The first step in the examination of the thymus in the patients with immunological disorders is to delineate the thymic outline by means of pneumomediastinography. Then a thymectomy via the suprasternal notch, occasionally combined with a parasternal incision, is performed. The lymphoid folliclle formations, which may serve as a parameter of immunological disorders, were found in some $74 \%$ in the removed thymuses of the patients with myasthenia gravis. Follow-up observations ranging between 1 and 15 years after operation revealed complete recovery in $27(24 \%)$, significant improvement in $47(43 \%)$ and moderate improvement in $21(19 \%)$ out of the 110 myasthenia gravis patients with nontumorous thymic abnormalities. Pneumomediastinography is also very useful to discover the small "occult thymoma" which is hardly ever found in the simple roentgenological examination. The lymphoid follicle formations in the removed thymuses were found in $16(43 \%)$ out of 37 patients with Behçet syndrome. In Behçet syndrome the recurrence and progression of oral and genital lesions were markedly suppressed after thymectomy. In high incidence lymphoid follicle formations were observed in the removed thymuses of patients with ulcerative colitis, chronic thyroiditis, systemic lupus erythematosus, autoimmune hemolytic anemia and Sjögren syndrome.
\end{abstract}

Key words: thymus, pneumomediastinography, mediastinoscopy, myasthenia gravis, autoimmune diseases.

(Received 13 September 1979)

The thymus had been a mysterious organ until the beginning of the present century, when its origin and structure began to be clarified. Still, its functional significance has remained unknown. Studies in immunity and its allied fields have disclosed that the thymus plays an important part as one of the central hymphoid tissues in immune regulation. From this point of view, the study of the thymus and its abnormalities have become more significant especially in the patients with immune disorders.

Introduction of mediastinoscopy devised by Carlens (1959) made it possible to examine the superior mediastinum directly, and to make biopsies of the thymic tumors in the retrosternal area. Moreover, it has become a matter of daily practice to extirpitate the 
whole thymus via the same incision in the patients with nontumorous thymic abnormalities (Akakura, 1965).

The present paper reports the method of study in such thymic abnormalities that are difficult to discover with routine X-ray examination, and the surgical procedure for removal of the nontumorous thymus and small "occult thymoma". Also the significance of thymectomy for the patients with autoimmune disorders will be discussed.

\section{Clinical examinations of thymic abnormalities}

The first step in examining the thymus is to delineate the thymic shadow with pneumomediastinography in the patients in whom the thymic abnormalities are suspected as often encountered in autoimmune diseases. After roentgenological examination, the patient is prepared for the thymectomy through the incision in the suprasternal notch, occasionally combined with parasternal incision (Table 1).

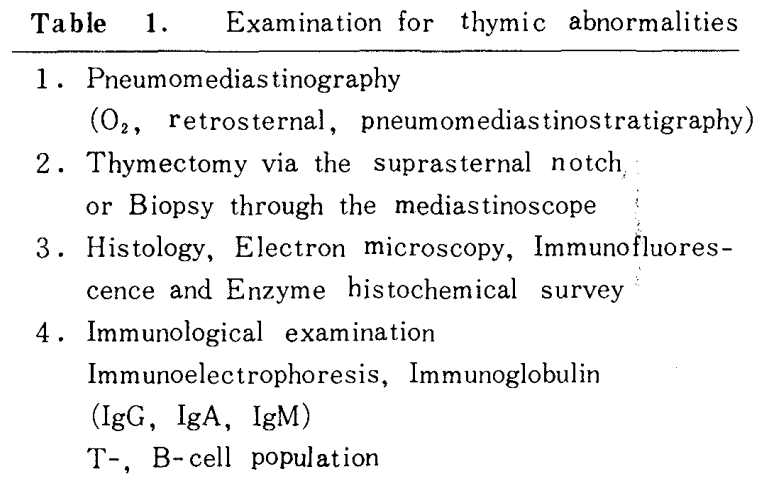

A. Pneumomediastinography (Fig. 1., 2a, b., 3a, b.)

It is usually difficult to detect a residue or hypertrophy of the thymus by means of a simple chest X-ray examination in patients with nontumorous thymic abnormalities. Therefore to examine the thymus in such a patient, pneumomediastinography or more particularly, pneumomediastinostratigraphy is initially performed by infusion with oxygen gas via the retrosternal region.

The instruments and materials required for pneumomediastinography include a puncture needle (lumbar or Cathelin's needle), vinyl or rubber tubing, a 100-ml syringe and a three-way stopcock as well as local anaesthetics, disinfectants and oxygen gas. 


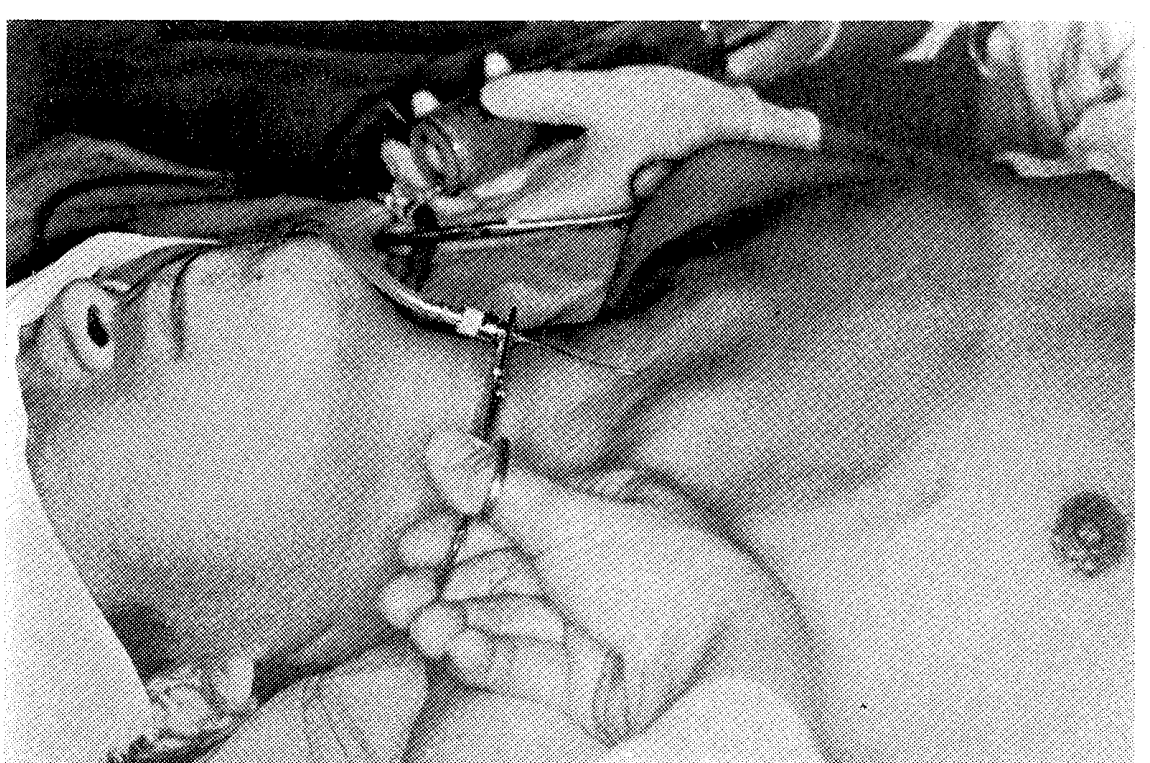

Fig. 1. Photograph showing the situation of pneumomediastinum through the retrosternal route.

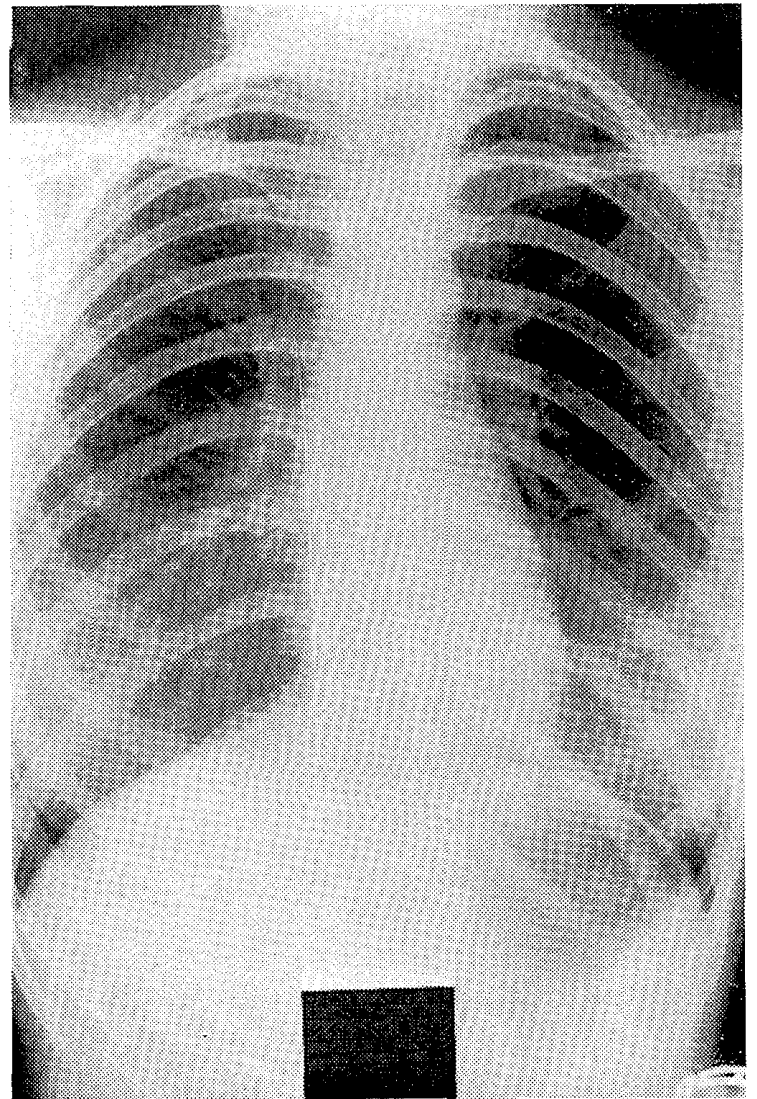

Fig. 2a. Roentgenogram of the chest of a patient with myasthenia gravis (22 year-old, female); no abnormal findings of the mediastinum were noted in a classical roentgenogram of the patient with nontumorous thymic abnormalities.

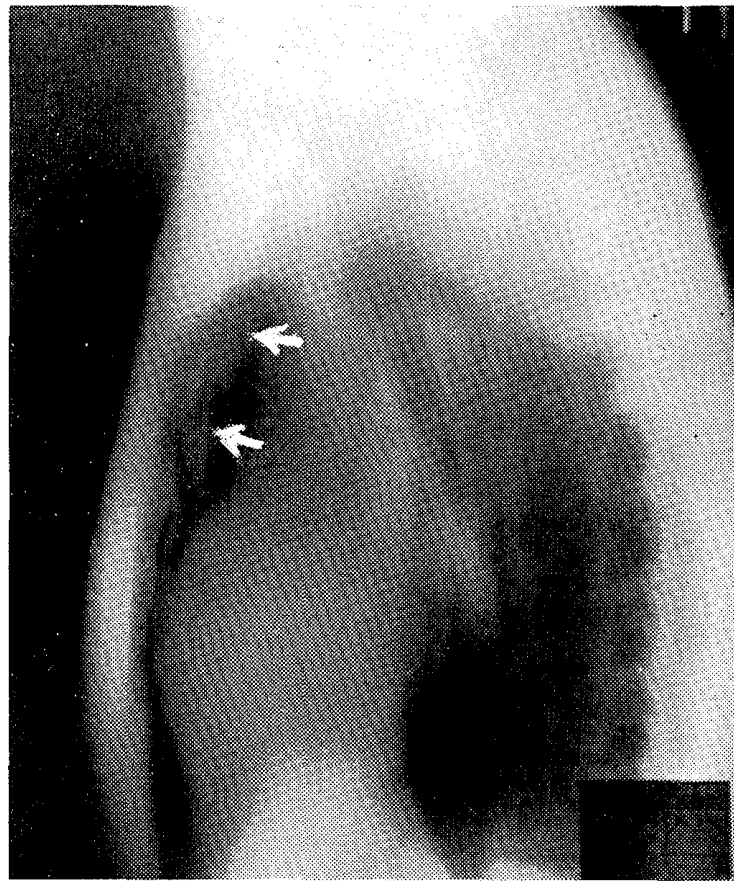

Fig. 2b. Thymus shadow in the pneumomediastinography after infusion of oxygen gas; nontumorous hypertrophy or residue of the thymus is demonstrated (arrow) after infusing $200 \mathrm{ml}$ oxygen gas. 


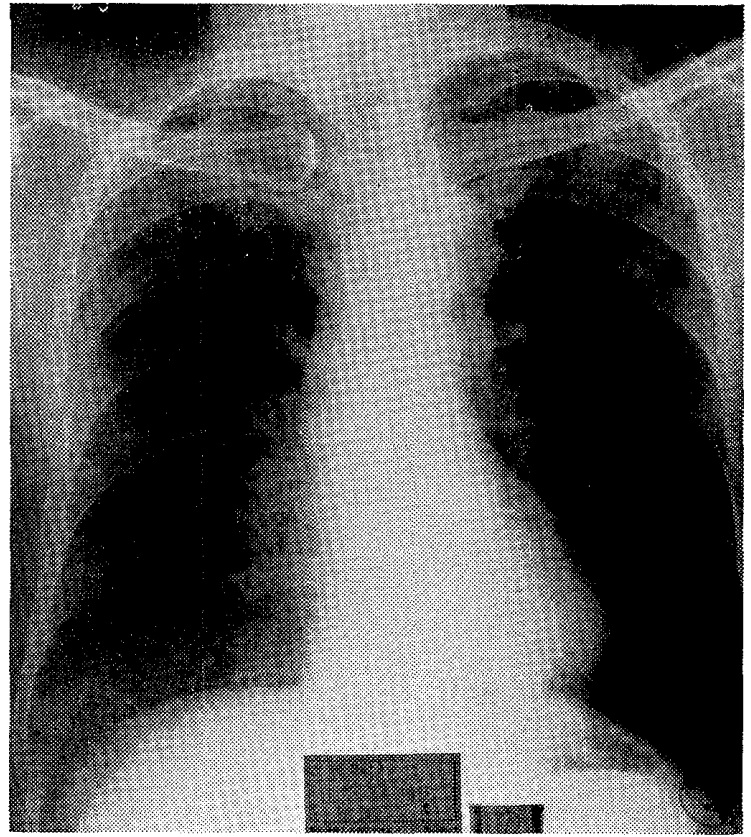

Fig. 3a. Roentgenogram of the chest of a patient with myasthenia gravis (61 year-old, male); no abnormal findings of the mediastinum was observed in this patient with a small thymic tumor.

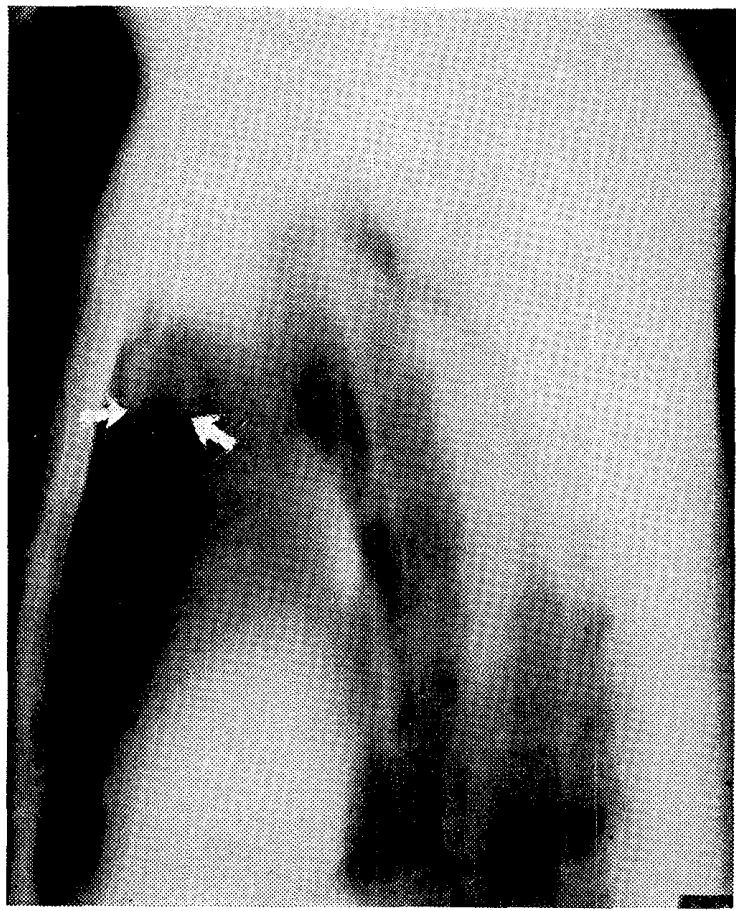

Fig. 3b. Thymus shadow with small thymoma in the pneumomediastinograph in the same patient; the shadow of tumorous portion (arrow) of the thymus is demonstrated as well as the nontumorous portion.

B. Technique for removal of the nontumorous thymus and/or small "occult thymoma" (Fig. 4, 5, 6, 7)

Under general anesthesia with endotracheal intubation, the patient is prepared for nonthoracotomic thymectomy via the suprasternal notch in the supine position.

After thymectomy, the area is throughly examined with a mediastinoscope to check for any hemorrhage. Moreover, if the thymus is situated in the anterior lower mediastinum, the parasternal incision is made, with the aid of a mediastinoscope, to perform mediastinal dissection and to remove the lower portion of the thymus. This procedure makes it easy to extirpitate the small "occult thymoma" with the nontumorous portion of the thymus.

Biopsy specimens were also obtained from the thyroid, sternocleidomastoid muscle and mediastinal lymph nodes through the same incision.

If the thymus exhibits any tumorous change with infiltration or adhesion to the surrounding tissues, biopsy should be performed by or with the aid of a mediastinoscope.

\section{Histopathological examination (Fig. 8, 9)}

The totally removed thymus and biopsy specimens of the thyroid, muscle and mediastinal lymph nodes obtained concomitantly are placed immediately in approximate fixatives 


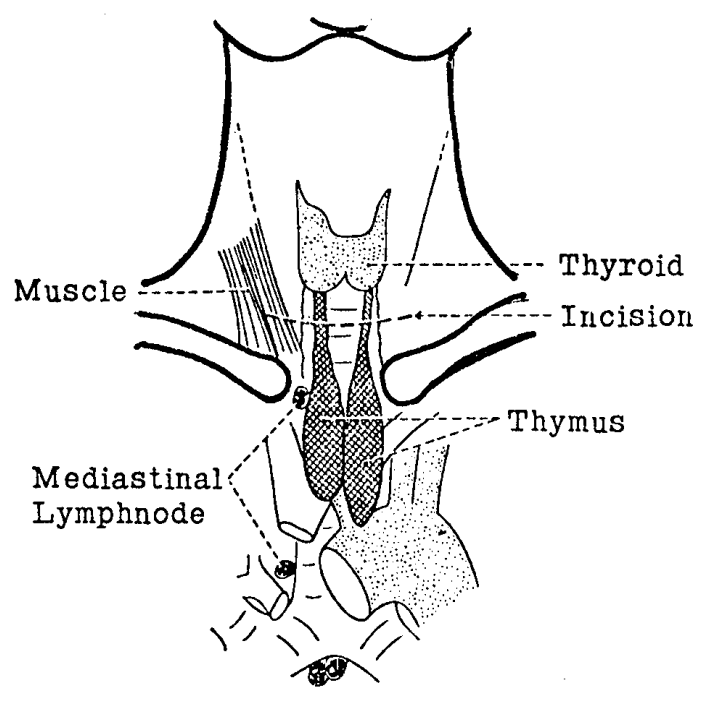

Fig. 4. Location of biopsy with thymectomy via the suprasternal notch; thyroid, muscle and mediastinal lymph nodes are partially resected as biopsy through the same incision (YoshimatsuTsuchiya, 1965).

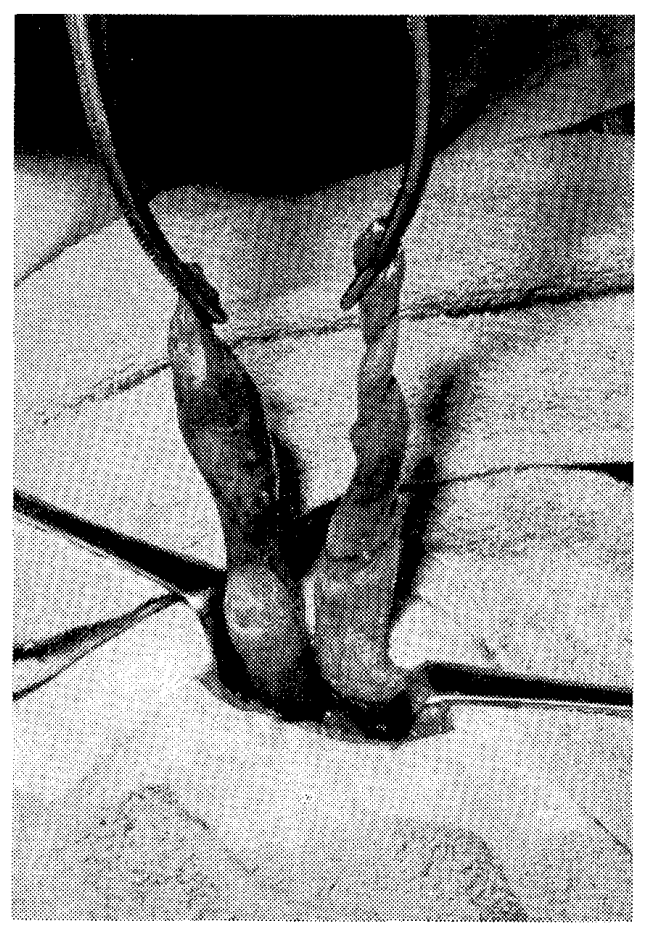

Fig. 6. Photograph of thymectomy via the suprasternal notch; in a majority of cases whose thymuses may be demonstrated only by mediastinography, the thymus is able to be excised through $4-5 \mathrm{~cm}$ transverse incision in the suprasternal notch.

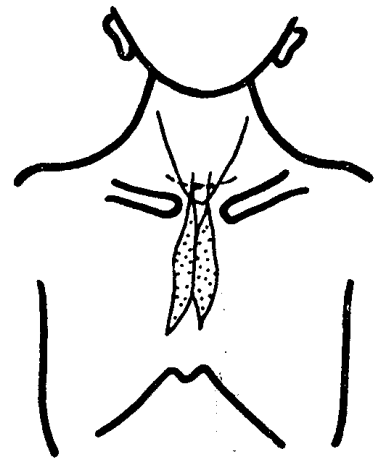

a

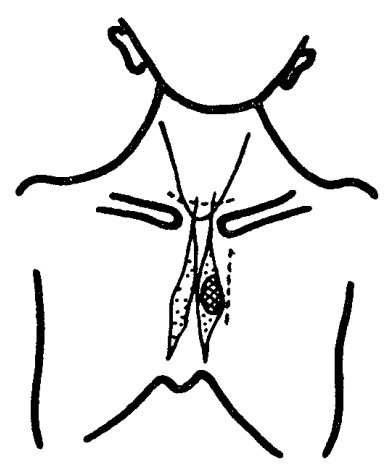

b
Fig. 5. Incision.

a. Incision in the suprasternal notch.

b. Transverse incision in the suprasternal notch and longitudinal incision on the parasternal region.

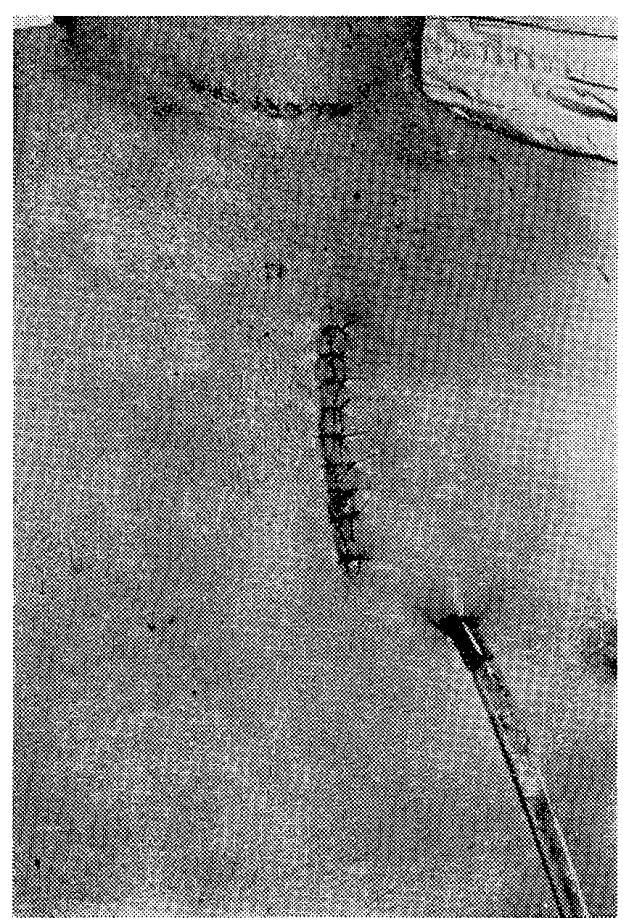

Fig. 7. Photograph showing the situation after thymectomy through the suprasternal and left parasternal incisions in a myasthenia gravis patient (23 year-old, male) with a small thymoma. 
and submitted for further processing to facilitate photomicroscopic and electronmicroscopic examinations as well as immunofluorescence and enzyme histochemical examinations.

D. Immunological examination

Samples of blood are drawn from the patients before and after thymectomy for immunological study. Sera are separated and subjected to immunophoretic analyses and

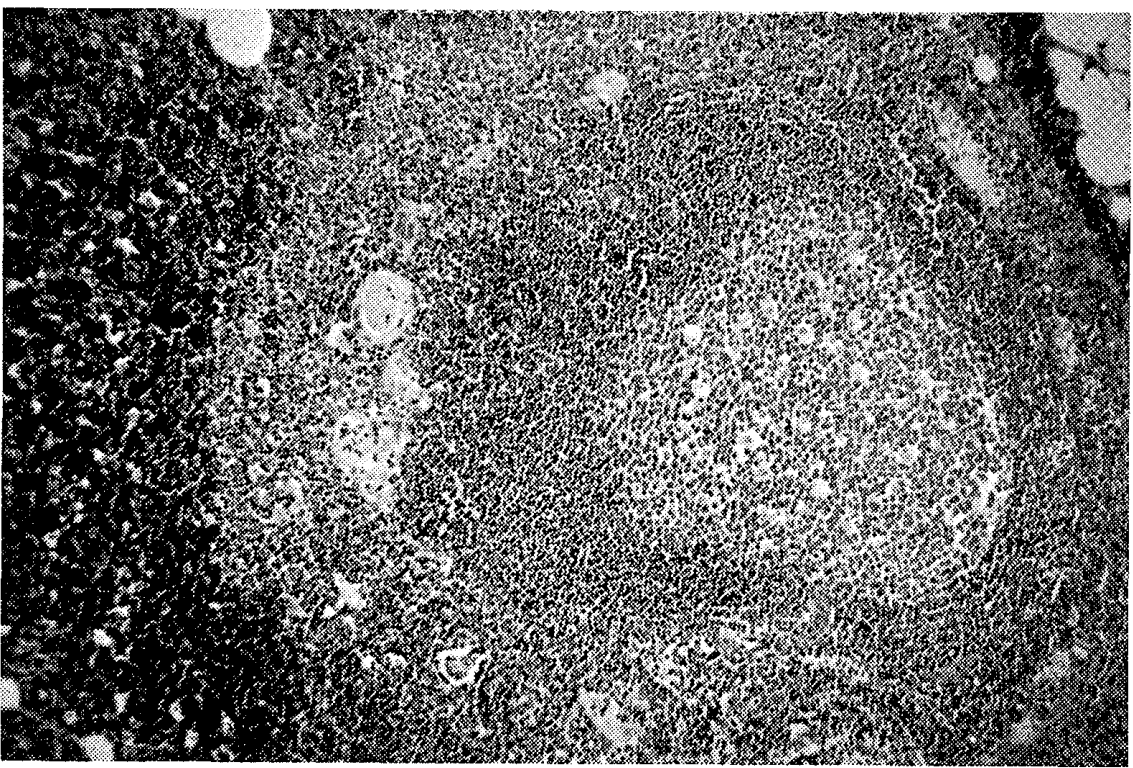

Fig. 8. Section of the removed thymus in a patient with myasthenia gravis (16 year-old, female); note the lymphoid follicle formation with germinal center in the medulla, Hassall's corpuscles are scattered around the follicle.

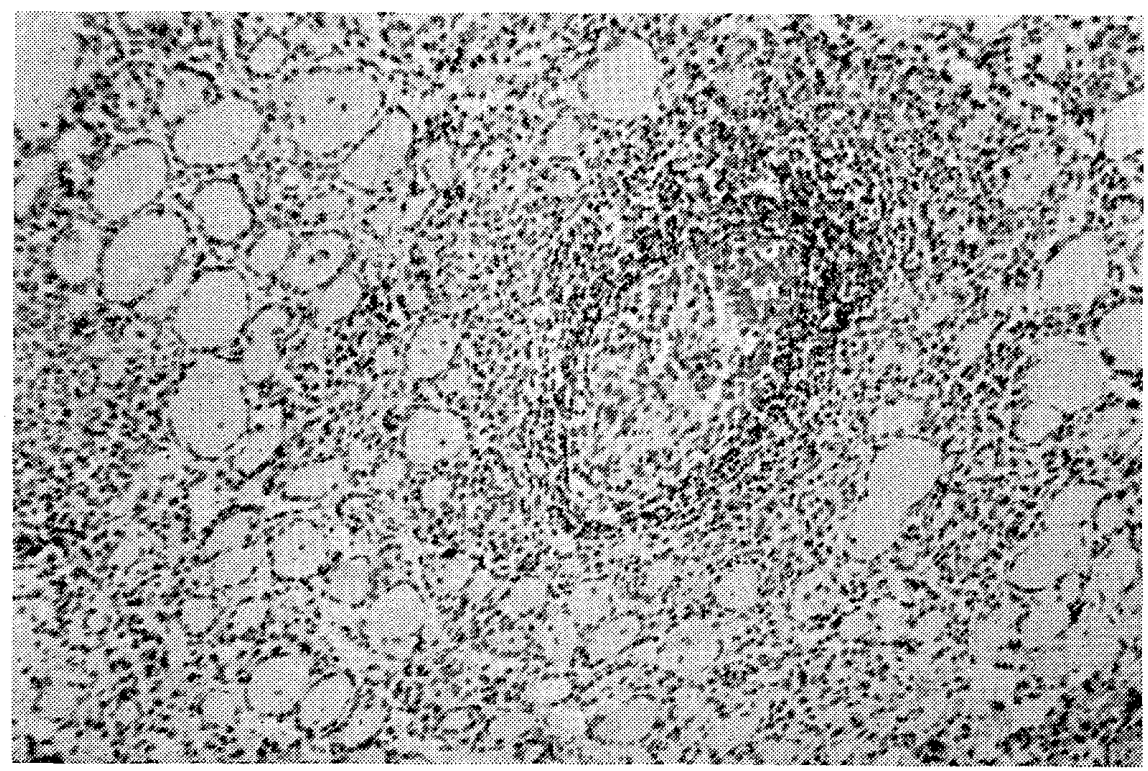

Fig. 9. Section of the thyroid specimen resected as biopsy; lymphoid follicle formation was noted in the same patient as commonly observed in Hashimoto's disease. 
examined for their immunoglobulin patterns. Each case is also assessed as to serum colloid reactions, lymphocytic count of the peripheral blood, endocrine functions, serum electrolyte levels before and after thymectomy.

Examination of immunological response of nude mice grafted with resected thymus and thymoma from patients with autoimmune diseases has also been done.

\section{Results and significance of thymectomy via suprasternal notch and/or parasternal incision in the patients with autoimmune diseases}

\section{A. Patients studied (Table 2, 3)}

We have performed 250 thymectomies on patients with myasthenia gravis, Behçet syndrome, ulcerative colitis, thyroid diseases, systemic lupus erythematosus and other various autoimmune diseases.

B. Myasthenia gravis with nontumorous thymic abnormalities (Table 4, 5, 6)

One-hundred and eighteen myasthenia gravis patients with nontumorous thymic abnormalities underwent a thymectomy via the suprasternal notch.

In the removed thymuses, lymphoid follicle formations were found in some $74 \%$, and in the thyroid resected as biopsy lymphoid follicle formations were found in $15 \%$.

Follow-up studies have been done at our clinic 6 months to 15 years after thymectomies. The patients were divided into 4 groups by the classification of Perlo et al. (1966). By their criteria, the prognoses were "completely recovered" in $27(24 \%)$ of 110 patients studied, and "significantly improved" in $47(43 \%) . \quad$ In addition, there

\begin{tabular}{|c|c|c|}
\hline Table & \multicolumn{2}{|c|}{$\begin{array}{l}\text { Cases of thymectomy via supraste- } \\
\text { rnal notch and/ or through paraster- } \\
\text { nal incision with mediastinoscopic } \\
\text { examination }\end{array}$} \\
\hline \multirow{3}{*}{\multicolumn{2}{|c|}{$\begin{array}{l}\text { Myasthenia gravis } \\
\qquad \begin{array}{l}\text { without thymoma } \\
\text { with occult thymoma }\end{array}\end{array}$}} & 130 \\
\hline & & 118) \\
\hline & & 12) \\
\hline \multicolumn{2}{|c|}{ Thyroid disease } & 20 \\
\hline \multicolumn{2}{|c|}{ Behçet syndrome } & 28 \\
\hline \multicolumn{2}{|c|}{ Systemic lupus erythematosus (SLE) } & 6 \\
\hline \multicolumn{2}{|c|}{ Autoimmune hemolytic anemia (AIHA) } & 3 \\
\hline \multicolumn{2}{|c|}{ Ulcerative colitis } & 28 \\
\hline \multicolumn{2}{|c|}{ Sjögren syndrome } & 1 \\
\hline \multicolumn{2}{|c|}{ Primary myxedema } & 4 \\
\hline \multicolumn{2}{|c|}{ Pune red cell anemia } & 2 \\
\hline \multicolumn{2}{|c|}{ Lupoid hepatitis } & 2 \\
\hline \multicolumn{2}{|c|}{ Occlusion of circulation of Willis } & 2 \\
\hline \multicolumn{2}{|c|}{ Burger's disease } & 3 \\
\hline \multicolumn{2}{|c|}{ Primary hyperparathyroidism } & 5 \\
\hline \multicolumn{2}{|c|}{ Sarcoidosis } & 4 \\
\hline \multicolumn{2}{|c|}{ Others } & 12 \\
\hline
\end{tabular}


Table 3. Number of subjects and incidence of lymphoid follicle formations in the removed thymus and the thyroid resected as biopsy

\begin{tabular}{|c|c|c|c|c|c|}
\hline \multirow{3}{*}{ Disease } & \multirow{3}{*}{$\begin{array}{c}\text { No. } \\
\text { cases } \\
\text { studied }\end{array}$} & \multicolumn{2}{|c|}{ Thymus } & \multicolumn{2}{|c|}{ Thyroid } \\
\hline & & \multicolumn{2}{|c|}{$\begin{array}{l}\text { Lymphoid follicle } \\
\text { formation }\end{array}$} & \multicolumn{2}{|c|}{$\begin{array}{c}\text { chr. lymphoid } \\
\text { thyroiditis }\end{array}$} \\
\hline & & Case & $(\%)$ & Case & $(\%)$ \\
\hline Myas thenia gravis & 110 & 81 & $(74)$ & 34 & (31) \\
\hline Behçet syndrome & 37 & 16 & $(43)$ & 4 & (11) \\
\hline Ulcerative colitis & 28 & 12 & $(43)$ & 3 & (11) \\
\hline Hashimoto thyroiditis & 10 & 4 & $(40)$ & 10 & $(100)$ \\
\hline SLE & 10 & 6 & $(60)$ & 2 & (20) \\
\hline Sjögnen syndnome & 2 & 2 & $(100)$ & 1 & (50) \\
\hline AIHA & 3 & 2 & (67) & 1 & (33) \\
\hline Total & 200 & 123 & $(62)$ & 55 & (28) \\
\hline Non-autoimmune disease & 63 & 3 & $(4.8)$ & & \\
\hline
\end{tabular}

\begin{tabular}{|c|c|c|c|c|c|}
\hline \multirow{3}{*}{$\begin{array}{l}\text { Age } \\
1-10\end{array}$} & 4. & Myastheria gravis & & , Sex & \multirow{3}{*}{ 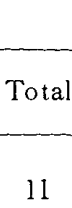 } \\
\hline & \multicolumn{2}{|c|}{$\begin{array}{l}\text { Patients with nontumorous } \\
\text { thymic abnormalities }\end{array}$} & \multicolumn{2}{|c|}{$\begin{array}{l}\text { Patients with } \\
\text { "Occult" thymoma }\end{array}$} & \\
\hline & $\underset{3}{\operatorname{male}}$ & $\underset{8}{\text { female }}$ & male & female & \\
\hline $11-20$ & 5 & 10 & & & 15 \\
\hline $21-30$ & 4 & 42 & 1 & & 47 \\
\hline $31-40$ & 3 & 25 & 1 & 1 & 30 \\
\hline $41-50$ & 3 & 9 & 2 & 2 & 16 \\
\hline $51-60$ & 1 & 3 & 2 & 2 & 8 \\
\hline $61-70$ & & 1 & 1 & & 2 \\
\hline $71-$ & 1 & & & & 1 \\
\hline Total & 20 & 98 & 7 & 5 & 130 \\
\hline
\end{tabular}

were 21 "moderately improved" patients (19\%) who were able to enjoy a normal life but still required continuation of medical treatment.

Thymectomy thus offered benefits eventually to 95 cases $(86 \%)$ of the myasthenia gravis patients with nontumorous thymic abnormalities.

C. Myasthenia gravis with "occult thymoma" (Table 7)

Extensive examination of thymic changes with pneumomediastinography or with mediastinoscopy revealed a small "occult thymoma" in the patients suspected of having thymic abnormalities.

Twelve myasthenia gravis patients were found to have small "occult thymoma". The size of these tumors was 2 to $6 \mathrm{~cm}$ long and 1 to $4 \mathrm{~cm}$ wide.

In future, it is hoped that the thymus with tumorous change will be found and removed completely at an earlier stage. 


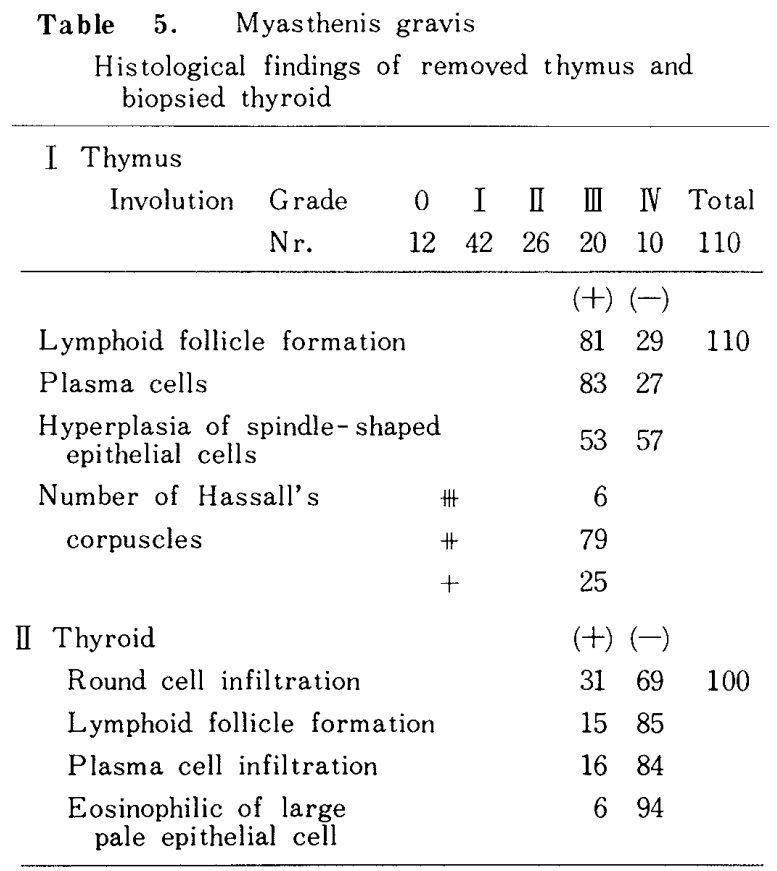

Table 6. Myasthenia gravis

- Results of thymectomy via the suprasternal notch in myasthenia gravis patients with nontumorous thymic abnormalities -

\begin{tabular}{|c|c|c|c|c|c|c|}
\hline \multirow[b]{2}{*}{ Group } & \multirow{2}{*}{$\begin{array}{l}\text { Number } \\
\text { of } \\
\text { patients }\end{array}$} & \multicolumn{3}{|c|}{ Good } & \multirow{2}{*}{$\begin{array}{c}\text { Un- } \\
\text { changed }\end{array}$} & \multirow[b]{2}{*}{ Dead } \\
\hline & & $\begin{array}{l}\text { Completely } \\
\text { recovered }\end{array}$ & $\begin{array}{l}\text { Significantly } \\
\text { improved }\end{array}$ & $\begin{array}{l}\text { Moderately } \\
\text { improved }\end{array}$ & & \\
\hline (I) & 17 & 8 & 6 & 3 & & \\
\hline$\left(\begin{array}{ll}\text { II } & \mathrm{A}\end{array}\right)$ & 33 & 7 & 20 & 4 & & 2 \\
\hline$\left(\begin{array}{ll}\text { II } & \mathrm{B}\end{array}\right)$ & 44 & 11 & 15 & 10 & 1 & 7 \\
\hline ( III) & 5 & 1 & 2 & & & 2 \\
\hline (IV) & 11 & & 4 & 4 & 1 & 2 \\
\hline Total & $\begin{array}{c}110 \\
(100 \%)\end{array}$ & $\begin{array}{c}27 \\
(24 \%)\end{array}$ & $\begin{array}{c}47 \\
(43 \%)\end{array}$ & $\begin{array}{c}21 \\
(19 \%)\end{array}$ & $\begin{array}{c}2 \\
(2 \%)\end{array}$ & $\begin{array}{c}13 \\
(12 \%)\end{array}$ \\
\hline
\end{tabular}

Severity and type (Perlo, Osserman 1966)

$\begin{array}{lll}\text { I } & \text { Ocular } \\ \text { II } & \text { A } & \text { Generalized mild } \\ \text { II } & \mathrm{B} & \text { Generalized moderately severe } \\ \text { III } & & \text { Acute fulminating } \\ \text { IV } & & \text { Late severe }\end{array}$

\section{Behçet syndrome (Table 8, 9)}

Behçet syndrome shows many characteristic signs and symptoms. The etiology of Behçet disease is yet unknown, but recently some dysimmune factors have been found in peripheral blood or intestinal tissues.

The patients with Behçet syndrome were divided into 3 groups according to previous 
Table 7. Myasthenia gravis patients with "occult thymoma”

\begin{tabular}{|c|c|c|c|c|c|c|c|}
\hline \multicolumn{4}{|c|}{ male } & \multicolumn{4}{|c|}{ female } \\
\hline $\begin{array}{l}\text { Case } \\
\text { age }\end{array}$ & Severity & $\begin{array}{l}\text { Examination } \\
\text { of thymoma }\end{array}$ & $\begin{array}{l}\text { Situation \& Size } \\
\text { of tumor }(\mathrm{cm})\end{array}$ & $\begin{array}{l}\text { Case } \\
\text { age }\end{array}$ & Severity & $\begin{array}{l}\text { Examination } \\
\text { of thymoma }\end{array}$ & $\begin{array}{l}\text { Situation \& Size } \\
\text { of tumor }(\mathrm{cm})\end{array}$ \\
\hline $\begin{array}{r}\text { 1. Y.S. } \\
28\end{array}$ & II $\mathrm{B}$ & $\mathrm{PMG}$ & $5.4 \times 3.5$ & 1. K.K. & IV & $\begin{array}{l}\text { IPMG } \\
\text { IOperation }\end{array}$ & $5.0 \times 3.5$ \\
\hline $\begin{array}{r}\text { 2. T.S. } \\
38\end{array}$ & II $\mathrm{A}$ & Operation & $2.0 \times 1.0$ & $\begin{array}{c}\text { 2. U.M. } \\
49\end{array}$ & IV & $\begin{array}{l}\text { IPMG } \\
\text { |Operation }\end{array}$ & $4.5 \times 3.0$ \\
\hline $\begin{array}{c}\text { 3. T.M. } \\
42\end{array}$ & IV & $\begin{array}{l}\text { PMG } \\
\text { Operation }\end{array}$ & $3.0 \times 3.0$ & $\begin{array}{r}\text { 3. M.I. } \\
51\end{array}$ & II A & PMG & $6.0 \times 4.0$ \\
\hline 4. T.I. & IV & $\begin{array}{l}\text { PMG } \\
\text { Operation }\end{array}$ & $4.0 \times 4.0$ & $\begin{array}{c}\text { 4. .N. } \\
53\end{array}$ & IV & PMG & $5.0 \times 4.0$ \\
\hline $\begin{array}{r}\text { 5. K.H. } \\
58\end{array}$ & II $\mathrm{B}$ & Operation & $4.5 \times 4.5$ & 5. S.H. & II B & PMG & $4.0 \times 3.0$ \\
\hline $\begin{array}{r}\text { 6. S. S. } \\
60\end{array}$ & II $\mathrm{A}$ & $\left\{\begin{array}{l}\text { PMG } \\
\text { Operation }\end{array}\right.$ & $3.0 \times 3.0$ & \multirow{2}{*}{\multicolumn{4}{|c|}{$\begin{array}{l}\text { PMG: Pneumomediastinography } \\
\text { Type and Severity } \\
\text { II A : generalized mild } \\
\text { II B } \text { generalized moderately severe } \\
\text { III } \vdots \text { acute fulminating } \\
\text { IV : late severe }\end{array}$}} \\
\hline $\begin{array}{r}\text { 7. S.E. } \\
70\end{array}$ & II $\mathrm{A}$ & Operation & $\Rightarrow=4.0 \times 3.5$ & & & & \\
\hline
\end{tabular}

systemic administration of corticoid.

In two groups (A and $B$ ) in which no treatment was given or steroid treatment had been discontinued 3 months prior to surgery, $15(62 \%)$ out of 24 patients had lymphoid follicle formations in the removed thymuses.

Twenty two $(79 \%)$ out of 28 patients had significant improvement and five (18\%), moderate improvement after surgery. Recurrence or progression of oral and genital mucosal lesions were markedly suppressed following thymectomy.

E. Ulcerative colitis (Table 10, Fig. 10)

In recent years, it has been reported that ulcerative colitis was rather frequently complicated with myasthenia gravis, Hashimoto's disease and systemic lupus erythmatosus. This fact and the usual therapeutic effectiveness of corticoid in this disease have directed general attention to its autoimmune aspect. Thymectomy via the suprasternal notch was performed on 28 patients with ulcerative colitis. The lymphoid follicle formations in the extirpitated thymuses were found in $12(43 \%)$ out of 28 patients.

A comparison was made of the clinical prognoses of thymectomized and nonthymectomized patients. In $91 \%$, the thymectomized patients have been kept in "inactive" conditions.

F. Others (Table 11, 12, 13)

The histological studies were also done in the removed thymuses as well as simultane- 
Table 8. Behçet syndrome. Histological findings of removed thymus and biopsied thyroid

\begin{tabular}{|c|c|c|c|c|}
\hline \multirow[b]{2}{*}{$\begin{array}{l}\text { Steroid administ- } \\
\text { ration }\end{array}$} & \multirow[b]{2}{*}{ Case } & \multicolumn{2}{|c|}{ Thymus } & \multirow{2}{*}{$\begin{array}{c}\text { Thyroid } \\
\begin{array}{l}\text { Lymphoid } \\
\text { thyroiditis }\end{array}\end{array}$} \\
\hline & & $\begin{array}{l}\text { Lymphoid follicle } \\
\text { formation }\end{array}$ & $\begin{array}{l}\text { Degenerative } \\
\text { change in } \\
\text { epithelial } \\
\text { elements (cells) }\end{array}$ & \\
\hline A. Nontreated & 14 & 7 & 4 & 3 \\
\hline B. Discontinued & 10 & 8 & 2 & 0 \\
\hline $\begin{array}{l}\text { C. Continued } \\
\text { within } 3 \\
\text { months before } \\
\text { operation }\end{array}$ & 13 & 1 & 11 & 1 \\
\hline Total & 37 & 16 & 17 & 4 \\
\hline
\end{tabular}

Table 9. Behçet syndrome. Clinical prognosis after thymectomy

\begin{tabular}{|c|c|c|c|c|}
\hline $\begin{array}{c}\text { Group } \\
\text { steroid admin. }\end{array}$ & $\begin{array}{l}\text { Number of } \\
\text { patients }\end{array}$ & $\begin{array}{l}\text { Significantly } \\
\text { improved }\end{array}$ & $\begin{array}{l}\text { Moderately } \\
\text { improved }\end{array}$ & $\begin{array}{c}\text { Un- } \\
\text { changed }\end{array}$ \\
\hline A. Nontreated & 14 & 12 & 1 & 1 \\
\hline B. Discontinued & 4 & 3 & 1 & \\
\hline $\begin{array}{l}\text { C. Continued } \\
\text { within } 3 \text { months } \\
\text { before operation }\end{array}$ & 10 & 7 & 3 & \\
\hline Total & 28 & 22 & 5 & 1 \\
\hline
\end{tabular}

Table 10. Ulcerative colitis

Histological findings of removed thymus and biopsied thyroid

\begin{tabular}{|c|c|c|c|c|}
\hline \multirow{2}{*}{$\begin{array}{l}\text { Clinical } \\
\text { severity }\end{array}$} & & \multirow{2}{*}{$\begin{array}{c}\text { Number of } \\
\text { patients }\end{array}$} & Thymus & \multirow{2}{*}{$\begin{array}{l}\text { Thyroid } \\
\text { Lymphoid } \\
\text { thyroiditis }\end{array}$} \\
\hline & & & $\begin{array}{l}\text { Lymph follicle } \\
\text { formation }\end{array}$ & \\
\hline \multirow{2}{*}{ A. mild } & $\int$ male & 5 & 2 & \\
\hline & female & 1 & 1 & \\
\hline B moderately & $\int$ male & 6 & 1 & 1 \\
\hline D. severe & female & 10 & 5 & 2 \\
\hline \multirow{2}{*}{ C. severe } & f male & 3 & 1 & \\
\hline & female & 3 & 2 & \\
\hline Total & & 28 & .12 & 3 \\
\hline
\end{tabular}

ously resected thyroids, mediastinal lymph nodes and sternocleidomastoid muscles as biopsy in the patients with systemic lupus erythematosus, Sjögren syndrome, autoimmune hemolytic 


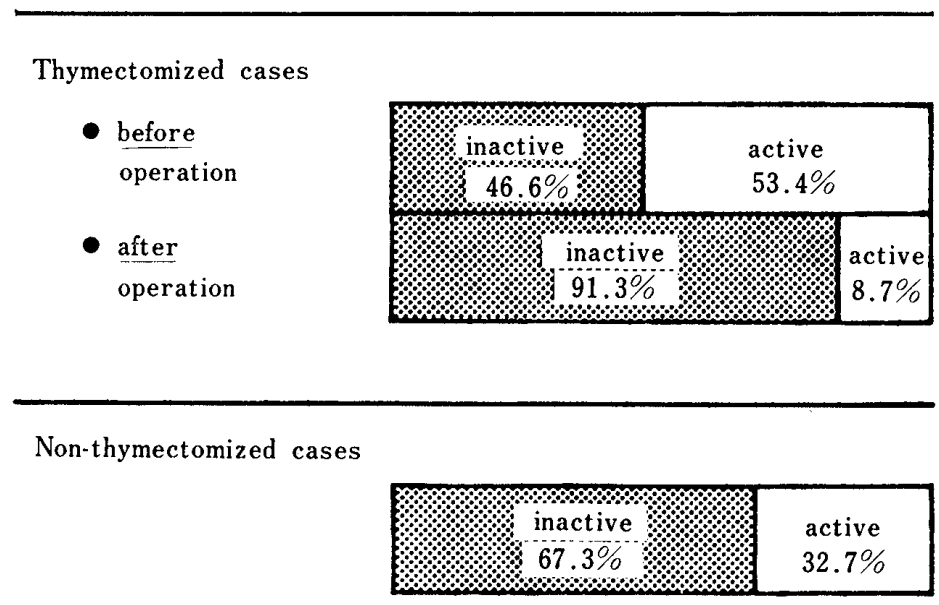

Fig. 10. Ulcerative colits. Clinical prognosis (per 100 patient years).

anemia and chronic thyroiditis (Hashimoto-type). The incidence of lymphoid follicle formations in the removed thymuses due to these diseases were $60 \%, 100 \%, 67 \%$ and $40 \%$, respectively.

\section{Discussion}

Recent progress in both functional and morphological study of the thymus together with the knowledge of transplantation immunity as well as cellular and tissue immunity has been remarkable especially during the last 20 years. These studies in immunity and its allied fields (Strauss et al., 1960; Miller, 1961; Burnet, 1962; Good \& Gabiersen, 1964; Oosterhius, 1964; Kobayashi et al., 1968; Papatestas et al., 1971; Souadjian et al., 1974; Shimabukuro et al., 1977) have disclosed that the thymus plays an important part as one of the central lymphoid tissues in immune regulation. From this viewpoint, the study of the thymus and its abnormalities has come to be more significant especially for the patients with immune disorders.

First reported by Willis in 1872, myasthenia gravis described by Erb (1879) as "a syndrome with the characteristic manifestation of marked weakness of muscle which may frequently proceed fatally" was defined as a clinical entity under the name of myasthenia gravis by Jolly (1895). Since the publication by Strauss et al. in 1960, the disease has come to be increasingly recognized as an autoimmune disease.

As for the relationship of the disease to thymic abnormality, Weigert (1901) found a thymic tumor at autopsy in a patient with myasthenia gravis and Blalock et al. (1939) reported the effectiveness of surgical removal of the thymona in the treatment of the disease. The technique discribed by Blalock was through the sternal splitting incision 
Table 11. Systemic lupus erythematosus (SLE)

Histological findings of removed thymus and biopsied thyroid

\begin{tabular}{|c|c|c|c|c|c|c|c|c|c|c|}
\hline \multirow{2}{*}{$\begin{array}{r}\text { Cas } \\
\text { ag }\end{array}$} & & & \multicolumn{5}{|c|}{ Thymusx } & \multirow[t]{2}{*}{ Thyroid } & \multirow[t]{2}{*}{ Muscle } & \multirow[t]{2}{*}{ N.B. } \\
\hline & & & 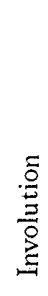 & 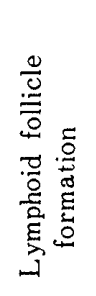 & $\begin{array}{l}\stackrel{\infty}{=} \\
\overline{0} \\
\stackrel{\infty}{\Xi} \\
0 \\
\frac{\infty}{a}\end{array}$ & 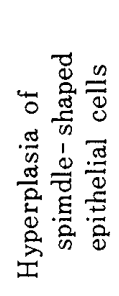 & 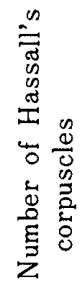 & & & \\
\hline R.N. & 21 & 우 & II & - & + & + & + & n.p. & Vasculitis & $\begin{array}{l}\text { Periportal cell } \\
\text { infiltration }\end{array}$ \\
\hline F.S. & 57 & 우 & II & \# & + & H & - & $\begin{array}{l}\text { Lymphoid } \\
\text { thyroiditis }\end{array}$ & n. p. & \\
\hline H. K. & 22 & 우 & I & - & - & - & + & n.p. & $\begin{array}{l}\text { Accumulation of } \\
\text { sarcolemmalnuclei }\end{array}$ & \\
\hline S.Y. & 20 & 우 & I & H & - & - & + & $\begin{array}{l}\text { Diffuse } \\
\text { thyroiditis }\end{array}$ & A trophy & \\
\hline$K . K$. & 15 & 우 & $I$ & \# & - & - & \pm & n. p. & Myositis & \\
\hline K.O. & 26 & 우 & II & + & - & + & H & n. p. & n.p. & \\
\hline
\end{tabular}

Table 12. Autoimmune hemolytic anemia (AIHA)

Histological findings of removed thymus and biopsied thyroid

\begin{tabular}{|c|c|c|c|c|c|c|c|c|c|}
\hline \multirow{2}{*}{$\begin{array}{r}\text { Case } \\
- \text { age } \\
\text { sex }\end{array}$} & & \multicolumn{5}{|c|}{ Thymus } & Thyroid & Muscle & $\begin{array}{l}\text { Mediastinal } \\
\text { Iymph node }\end{array}$ \\
\hline & & $\begin{array}{l}\stackrel{5}{\Xi} \\
\stackrel{\Xi}{\Xi} \\
\stackrel{\Xi}{\Xi}\end{array}$ & 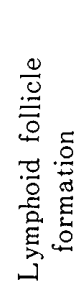 & 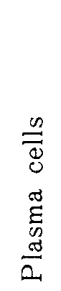 & 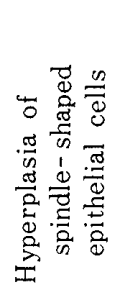 & 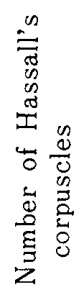 & & & \\
\hline A.T. 34 & 우 & IV & - & + & + & + & $\begin{array}{l}\text { Lymphoid } \\
\text { thyroiditis }\end{array}$ & n.p. & $\begin{array}{l}\text { Chronic } \\
\text { Iymphadenitis }\end{array}$ \\
\hline T.Z. 14 & 우 & I & + & + & - & + & $\begin{array}{l}\text { Lymphoid } \\
\text { thyroiditis }\end{array}$ & n.p. & \\
\hline T.S. 16 & 우 & II & + & - & - & + & Fibrosis & n. p. & \\
\hline
\end{tabular}

from the suprasternal fossa to the right third intercostal space. The first patient of thymectomy in Japan was reported by Tashioro (1940) and a number of thymectomized patients have thereafter been reported, all operated on using either the transthoracic or sternal splitting incision.

The surgical procedure we have hitherto adopted for removal of the thymus in patients with autoimmune diseases at Keio Universety Hospital and Kitasato Institute Hospital is "Thymectomy via the Suprasternal Notch" devised on the basis of our own experiences in 
Table 13. Chronic thyroiditis of Hashimoto type

\begin{tabular}{|c|c|c|c|c|c|c|c|c|c|c|}
\hline \multirow[t]{2}{*}{ Case } & \multicolumn{2}{|l|}{ Age } & \multirow[t]{2}{*}{ Complication } & \multicolumn{4}{|c|}{ Thymus } & \multirow[t]{2}{*}{ Thyroid } & \multirow[t]{2}{*}{ Muscle } & \multirow[t]{2}{*}{$\begin{array}{l}\text { Mediastinal } \\
\text { Lymphnodes }\end{array}$} \\
\hline & & & & 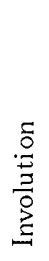 & 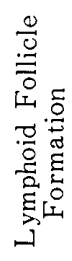 & 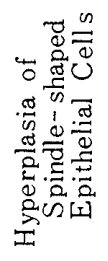 & 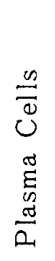 & & & \\
\hline I . T. & 23 & 우 & SLE & II & + & - & + & $\begin{array}{l}\text { Diffuse } \\
\quad \text { Thyroiditis }\end{array}$ & n.p. & \\
\hline $\mathrm{K} \cdot \mathrm{M}$. & 17 & 우 & $\begin{array}{l}\text { Myasthenia } \\
\text { Gravis }\end{array}$ & $\mathrm{I}$ & Ht & + & + & " & $\begin{array}{l}\text { Accumulation } \\
\text { of Sarcolem- } \\
\text { marrnurclei }\end{array}$ & $\begin{array}{l}\text { Chronic } \\
\text { Lymphadenitis }\end{array}$ \\
\hline$M . K$. & 36 & 우 & & III & - & - & + & $"$ & n.p. & \\
\hline A.I. & 40 & 우 & $\begin{array}{l}\text { Myasthenia } \\
\text { Gravis }\end{array}$ & III & \# & - & + & " & $\begin{array}{l}\text { Simple } \\
\text { A trophy }\end{array}$ & $\begin{array}{l}\text { Follicular } \\
\text { Hyperplasia }\end{array}$ \\
\hline Y.O. & 50 & 우 & & III & - & - & - & " & n.p. & \\
\hline A. A. & 51 & 우 & & V & - & - & - & $"$ & n. p. & \\
\hline N.S. & 52 & 우 & $\begin{array}{l}\text { Diabetes } \\
\text { Mellitus }\end{array}$ & II & + & - & - & " & n. p. & \\
\hline Y.M. & 54 & $\hat{\delta}$ & Fatty Liver & N & - & . H & + & " & n.p. & $\begin{array}{l}\text { Chronic } \\
\text { Lymphadenitis }\end{array}$ \\
\hline M.K. & 56 & $\hat{\delta}$ & & $\mathbb{N}$ & - & - & - & " & n.p. & \\
\hline I . S . & 73 & 우 & & III & - & + & + & " & n. $p$. & $\begin{array}{l}\text { Chronic } \\
\text { Lymphadenitis }\end{array}$ \\
\hline A. T. & 33 & 우 & $\begin{array}{l}\text { Myasthenia } \\
\text { Gravis }\end{array}$ & l & \# & - & + & $\begin{array}{l}\text { Focal } \\
\text { Thyroiditis }\end{array}$ & $\begin{array}{l}\text { Simple } \\
\text { A trophy }\end{array}$ & \\
\hline A. H. & 65 & 우 & Anemia & IV & - & - & + & " & n.p. & $\begin{array}{l}\text { Chronic } \\
\text { Lymphadenitis }\end{array}$ \\
\hline
\end{tabular}

removal of thymic cyst after mediastinoscopic examination of the superior mediastinum.

The introduction of mediastinoscopy devised by Carlens (1959) and the anatomical studies of mediastinum by Sarrazin (1971) made it possible to examine the superior mediastinum directly and to perform a biopsy of the thymic tumor. The removal of the whole nontumorous thymus through the suprasternal incisiosn is now common practice in our clinic.

A thymectomy was performed by our surgical technique on 250 patients, which included 237 with nontumorous thymic abnormalities and 13 with smaall "occult thymoma".

Kirschner et al. (1969) adopted the technique, too, as an operative procedure for the treatment of myashenia gravis at Mt. Sinai Hospital, New York, and emphasized the advantage of "Transcervical total thymectomy" and potential extension of its indications because of the considerable reduction in surgical stress as compared with other surgical techniques. Bibliographycally, the method of "thymectomy through the neck" is described by some 
plates of illustrations as an emergency surgical measure for tracheal stenosis in the "Thyroid and Thymus" by Crotti (1918).

With a view of studying thymic changes to clarify the etiology of autoimmune diseases, Gunn \& Michie (1965) performed a thymectomy through the neck, and Crile (1966) adopted the same surgical procedure as pretreatment for renal transplantation. These two examples emphasize the usefulness of this technique which facilitated removal of the thymus without opening the chest and with little surgical stress in patients with renal failure and poor general status.

Keynes (1949) described that complete or almost complete remission occured in $65 \%$ of a total of 120 operated myasthenia gravis cases with nontumorous thymic abnormalities. According to Schwab \& Leland (1953), 63\% of women without thymomas, who were thymetomized for thymic anomalies, displayed unquestionable symptomatic ameliolation. According to prolonged follow-up observations in numerous thymectomized patients of myasthenia gravis made by Eaton \& Clagett (1955), Osserman et al. (1958), Simpson (1958), Lange (1960), Perlo et al. (1966), Kuroshima (1966) and Shibouda et al. (1969), almost of the authors have confirmed effectiveness of the surgical treatment, and many reports communicated the common view that the prognosis after thymectomy of myasthenia gravis without thymoma is more favorable in women if operated on at relatively short periods after onset of the illness.

The one-hundred eighteen myasthenia gravis patients with nontumorous thymic abnormalities reported herein were all totally thymectomized via the suprasternal notch, occasionally combined with mediastinoscopic dissection through the parasternal incision. The patients ranged from 18 months to 74 years in age and the surgical measure proved to be positively practicable in a wide variety of patients from infants with ocular type of the disease to patients at older ages with the advanced severe type. Not a single surgical death occured. Thus thymectomy via the suprasternal notch is extremely advantageous over the conventional procedures of thymectomy by way of thoracotomy or sternal splitting incision.

The thymus which is detectable only by such a special examination as pneumomediastinography, Mizuno et al. (1976), can totally be removed by this procedure. However, if any tumorous change has been noted to exist in the thymus, biopsy should be undertaken with the aid of a mediastinoscope and removal of the thymic tumor done in combination with a parasternal incison and/or occasionally with thoracotomy.

Myasthenia gravis, as it is generally believed to represent an autoimmune disease, it is advised that biopsy specimens of the thyroid, muscles and mediastinal lymph nodes will be obtained through the same incion in the suprasternal notch and that histopathological diagnoses should be made with these specimens. (Yoshimatsu, 1965; Yoshimatsu et al., 1971, 1972)

Moreover, we have made it a rule to examine the thymus microscopically as to lymphoid follicle formation with germinal center which may serve as a parameter of immunological disorders. 
Twenty-eight patients with Behçet syndrome underwent thymectomy. The etiology of Behçet syndrome is yet unknown, but there are a variety of hypotheses; viral and/or bacterial infection, allergy and the autoimmunity. Recently Behçet syndrome has been studied from the standpoint of autoimmunity theory by Shimizu et al. (1965). The authors speculated that if Beçet syndrome is regarded as an autoimmune disease, the thymic abnormalities should be found in the patients with the disease as in other autoimmune diseases. Pneumomediastionography revealed an abnormally large thymic shadow in all patients with Behçet syndrome. Thus, the authors have carried out the clinical study, expecting that the thymectomy will be an efficient therapeutic means to suppress the progress of Behçet syndrome.

It is noteworthy that the lymphoid follicle formations were observed frequently in the removed thymus of the patients with Behçet syndrome, because it has been well recognized that the formations of lymphoid follicles in the thymus are found in various autoimmune disease such as myasthenia gravis and SLE (Habu et al., 1971).

The pathohistological studies in the removed thymuses and follow-up studies after thymectomy suggested that the thymic abnormalities play an important role in the etiology of Behçet syndrome and that thymectomy contributes greatly to the inhibition of the progress of this disease. (Tsuchiya et al., 1974).

Twenty eight patients with ulcerative colitis underwent thymectomy. This is one of the diseases the etiology of which is unknown, but the result of thymectomy gives some evidences to show the autoimmunity. (Tsuchiya, 1978). We have studied a possible role of the humorally and cellularly mediated immunological disorders and thymic abnormalities in mechanisms of its etiology.

The results of histological studies and follow-up studies after thymectomy in the patients with Sjögren syndrome or with autoimmune hemolytic anemia will also clarify to some extent the etiology of their complicated disorders and may elicit some better treatment.

\section{Conclusion}

Results and significance of thymectomy for 250 patients with autoimmune diseases, which include myasthenia gravis, Behçet syndrome, ulcerative cloitis and others, were studied.

The thymus delineated by means of pneumomediastinography was almost always successfully removed by our thymectomy via the suprasternal notch, occasionally combined with parasternal incision.

Thymectomy via the suprasternal notch was performed on a series of 118 patients with myasthenia gravis associated with nontumorous thymic abnormalities, 98 females and 20 males, ranging in ages from 18 months to 74 years. In the removed thymuses, lymphoid follicle formations were found in some $74 \%$ in high incidence as often encountered 
in autoimmune diseases. Follow-up observations, ranging from 1 year to 15 years after surgery, revealed complete recovery, significant and moderate improvement in 95 (86\%) of the 110 myasthenia gravis patients with nontumorous thymic abnormalities.

Pneumomediastinography is also very useful to discover the small "occult thymoma" which is hardly ever found in the classical roentgenological examination.

The immunological role of the thymus and the relationship between histological changes and clinical symptoms in those patients with myasthenia gravis, Behçet syndrome, ulcerative colitis and many other autoimmune diseases, must be studied to clarify their etiology and to establish the treatment.

Many problems concerning these thymic abnormalities will be settled by the cooperative efforts of basic scientists and clinical workers.

\section{Acknowledgement}

This study was supported (in part) by the Research Grant for the Intractable Diseases from the Ministry of Health and Welfare of Japan.

\section{References}

Akakura, I. (1965): Mediastinoscopy. XI Internat. Congr. Bronchoesophagology, Japan. pp. 6-9.

Blalock, A., Mason, M. F., Morgan, H. J. et al. (1939): Myasthenia gravis and tumors of the thymic region. Ann. Surg., 110: 544-561.

Burnet, F. M. (1962): Role of the thymus and related organs in immunity. Brit. Med. J., 2: 807-811.

Carlens, E. (1959): Mediastinoscopy; A method for inspection and tissue biopsy in the superior mediastinum. Dis. Chest, 36: 343-352.

Carlens, E., Johansson, L. \& Olsson, P. (1967): Mediastinoscopy auxiliary to thymectomy by the cervical route. Bronches, 17: 408-410. (in French)

Crile, G., Jr. (1966): Thymectomy through the neck. Surgery, 59: 213-21.5.

Crotti (1918, 1923): Surgical technic of thymectomy. In: Thyroid and Thymus. Lea \& Febiger.

Eaton, L. M. \& Clagett, O.T. (1955): Present status of thymectomy in treatment of myasthenia gravis. Amer. J. Med., 19: 703-717.

Erb, W. (1879): Zur Casuistik der bulbären Lähmunger: 3. Ueber einen neuen wahrscheinlich bulbären Symptomencomplex. Arch. Psychiatr., 9: 336-350.

Good, R.A. \& Gabliersen, A.E. (1964): Clinical considerations of the thymus in immunology. In: The Thymus in Immunology, Hoeber, New York. pp. 3-47.

Gunn, A., Michie, W. \& Irvine, W. J. (1964): The thymus in thyroid diseases. Lancet, II: 776777.

Gunn, A. \& Michie, W. (1965): Biopsy of thymus. Brit. J. Surg., 52: 957-963.

Habu, S., Kameya, T. \& Tamaoki, N. (1971): Thymic lymphoid follicles in autoimmune disease. I-Quantitative studies with special reference to myathenia gravis. Keio J. Med., 20: 45-56.

Jolly, F. (1895): Ueber myasthenia gravis pseudoparalytica. Berl. Klin. Wschr., 1: 1-7.

Kirschner, P. A., Osserman, K.E. \& Kark, A. E. (1969): Studies in myasthenia gravis - Transcervical total thymectomy-. JAMA, 209: 906-910.

Keynes, G. (1949): The results of thymectomy in myasthenia gravis. Brit. Med. J., 2: 611-616.

Kobayashi, T., Watanabe, H., Akiyama, T. et al. (1968): The Thymus-Basic and Clinical Studies. 
Igaku-Shoin, Tokyo. (in Japanese)

Kuroshima, S. (1966): Thymectomy for myasthenia gravis. Surg. Diag. \& Treat., 8: 1184-1192. (in Japanese)

Lange, M. (1960): The preparation for and the results of surgery in myasthenia gravis. Brit. J. Surg., 48: 285-291.

Miller, J.F.A.P. (1961): Immunological function of the thymus. Lancet, II: 748-794.

Mizuno, Y., Shimabukuro, K., Kurita, K. et al. (1976): Thymus abnormalities in ulcerative colitiscomparative study with other autoimmune diseases. Gastroenterologia Japonica, II: 208-214.

Oosterhius, H. J. (1964): Studies in myasthenia gravis: I. A clinical study of 180 patients. J. Neurol. Sci. , 1: 512-546.

Osserman, K. E., Kornfeld, P., Cohen, E. et al. (1958): Studies in myasthenia gravis. Arch. Int. Med., 102: 72-81.

Papatestas, A.E., Osserman, K.E. \& Kark, A.E. (1971): The relationship between thymus and oncogenesis. A study of the incidence of non-thymic malignancy in myasthenia gravis. Brit. J. Cancer, 4: 635-645.

Perlo, V.P., Poskanzer, D.C., Schwab, R.S. et al. (1966): Myasthenis gravis: Evaluation of treatment in 1, 355 patients. Neurology, 16: 431-439.

Sarrazin, R. (1971): Anatomical Background to Mediastinoscopy. In: Mediastinoscopy. Odense University Press. Denmark. pp. 6-10.

Schwab, R.S. \& Leland, C.C. (1953): Sex and age in myasthenia gravis as critical factors in incidence and remission. JAMA, 153: 1270-1273.

Shibouda, Y., Kagawa, K., Saji, K. et al. (1969): Effect of Thymectomy on Myasthenia Gravis. Proc. Myasthenia Gravis Symp. I: 73-76. (in Japanese)

Shimabukuro, K., Ono, A., Aiso, T. et al. (1977): Immunological responce of nude mice grafted with hyperplastic thymus and thymoma from autoimmune diseases. Proc. of the Second International Workshop on Nude Mice. University of Tokyo Press, Tokyo. pp. 275-286.

Shimizu, T., Katsuta, Y. \& Oshima, Y. (1965): Immunological studies on Behçet's syndrome. Ann. Rheum. Dis., 24: 494-500.

Simpson, J.A. (1958): An evaluation of thymectomy in myasthenia gravis. Brain, 81: 112-144.

Souadjian, J. V., Enriquez, P., Silverstein, M. N. et al. (1974): The spectrum of diseases associated with thymona. Arch. Int. Med., 134: 374-379.

Strauss, A. J.L., Seegal, B.C., Hsu, K. C. et al. (1960): Immunofluorescence demonstration of a muscle binding, complement fixing serum globulin fraction in myasthenia gravis. Proc. Soc. Exper. Biol. and Med., 105: 184-191.

Tashiro, K. (1940): Myasthenia gravis, A thymectomized case. J. Jap. Surg. Soci., 41: 398. (in Japanese)

Tsuchiya, M., Yoshimatsu, H., Habu, S. et al. (1974): Thymic hyperplasia and therapeutic approach of thymectomy in Behçet's syndrome. Biochm. and Exper. Biol., XI. n. 1, 47-52.

Tsuchiya, M. (1978): Clinical studies in ulcerative colitis. Keio Igaku, 55: 1-11. (in Japanese)

Weigert, C. (1901): Pathologisch-anatomischer Beitrag zur Erbsen Krankheit (Myasthenia gravis). Neurol. Centrable, 20: 597-601.

Yoshimatsu, H. (1965): The significance of thymus in surgical clinic-Thymectomy via the suprasternal notch-. Saishin-Igaku, 20:2758-2768. (in Japanese)

Yoshimatsu, H., Tsuchiya, M., Kamegaya, K. et al. (1971): Mediastinoscopy and Thymectomy via the Suprasternal Notch. In: Mediastinoscopy. Odense University Press, Denmark. pp. 94-96.

Yoshimatsu, H., Ikeda, T., Tsuchiya, M. et al. (1971): Mediastinoscopy and its application; Thymectomy via the suprasternal Notch. Keio J. Med., 20: 29-43.

Yoshimatsu, H., Ishihara, T., Ikeda, T. et al. (1972): Results and effectiveness of thymectomy via the suprasternal notch in myasthenia gravis. Keio J. Med., 21:215-233. 
胸腺異常の検索法並びに重症筋無力症その他自己免疫疾患例における胸腺剔出の意義

吉松博・石食義弥・村上勝・川原英之

产業察科大学第二外科学教室

要旨：胸腺に関する形態的、機能的研究はこの20年間に移植免疫をはじぬ細胞・組織免疫などの 分野からの学明とともに著しい進歩を遂げた。しかしヒト胸腺の免疫調節機構における重 要蔵器の一つとしての役劃や多くの免疫異常疾患との関連についてはな妳解すべき多く の問題が残されている。胸腺異常の臨称的検索法ならびに重症筋無力症をはじめとする各 種自己免疫疾患の病因解明或は治療法開発のため、胸腺剔出を行った 250 例につき検討し、 次の如き成績をえた。胸腺異常の形態的検查は臨床上レ線学的に縦陾充気法 (気縦隔) が 有用であり、重症筋算力症例においては剔出脸腚の約 75\%に自己免疫疾患例で高頻度に見 出されるリンパ㵽胞形成が見出された。胸腺剔出後 1 年から 15 年間にわたる観察で、そ

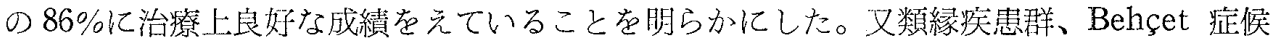

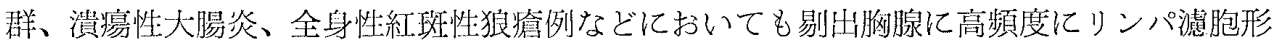
成を見出し、腧腺剔出後、症状の進展阻止を認め治療法の一助として有用な点も明らかに した。

産業医大誌，1 14 ) : 487-505 